\title{
Risk of prevalence of latent tuberculosis infection in health care workers-an idiographic meta-analysis from a Chinese perspective
}

\author{
Hai-Yan Guo $^{1 \#}$, Qian-Hong Zhong ${ }^{3 \#}$, Jie Zhou ${ }^{4}$, Zhi-Meng Zhao ${ }^{3}$, Xi-Lin Zhang ${ }^{3}$, Zhen-Huang Chen ${ }^{1,2}$, \\ Xin-Cai Qiu ${ }^{1,2}$, Zhi-Long $\mathrm{Wu}^{3}$ \\ ${ }^{1}$ Department of Respiratory Medicine, Nanhai Hospital Affiliated to Southern Medical University, Foshan, China; ${ }^{2}$ The Second School of Clinical \\ Medicine, Southern Medical University, Foshan, China; ${ }^{3}$ Department of Respiratory Medicine, The Fourth People's Hospital of Foshan (Tuberculosis \\ Control Center of Foshan), Foshan, China; ${ }^{4}$ Department of Respiratory Medicine, Foshan Hospital of TCM, Foshan, China \\ Contributions: (I) Conception and design: HY Guo, QH Zhong, ZL Wu; (II) Administrative support: ZH Chen, XC Qiu, ZL Wu; (III) Provision of \\ study materials or patients: None; (IV) Collection and assembly of data: HY Guo, QH Zhong; (V) Data analysis and interpretation: QH Zhong, ZM \\ Zhao; (VI) Manuscript writing: All authors; (VII) Final approval of manuscript: All authors. \\ "These authors contributed equally to this work. \\ Correspondence to: Dr. Zhi-Long Wu. Department of Respiratory Medicine, The Fourth People's Hospital of Foshan, No. 106, Jinyu South Road, \\ Foshan, China. Email: wuzlfs@163.com.
}

Background: China is one of the countries sharing the major burden of tuberculosis (TB) in the world. Health care workers (HCWs) are subject to a high risk of occupational latent tuberculosis infection (LTBI) an asymptomatic state of TB disease. However, the heterogenic composition of healthcare professionals in terms of nature of their work leads to the inconsistency in predicting the prevalence of LTBI amongst them. Furthermore, the global statistics do not account for the analysis conducted within the Chinese population. Our study reflects a systemic and epidemiological meta-analysis to investigate the risk of contracting LTBI by the HCWs of China.

Methods: A systematic review of the literature was performed to identify studies reporting LTBI prevalence or incidence among HCWs and a control groups in China. Risk of infection, as well as subgroup analysis was calculated by pooled effect estimates. Review Manager 5.0 was used to perform the meta-analyses.

Results: Twenty studies containing 9,654 HCWs met the inclusion criteria. The average prevalence of LTBI among HCWs was 51.5\%, ranging from 27.9-88.8\%. HCWs had a higher risk of prevalence of LTBI than the control groups [odds ratio (OR), 1.78, 95\% confidence interval (CI), 1.46-2.16]. In the subgroup analysis, the prevalence of LTBI in HCWs with respect to the control groups was observed to be highest in Eastern China (OR, 2.05; 95\% CI, 1.35-3.11). Furthermore, the pooled OR for LTBI was 1.90 and 1.65 separately from the results of the tuberculin skin test (TST) and the interferon-gamma release assay. Lastly, upon comparing the HCWs with the control groups from the community and the nosocomial source, it was observed that the pooled OR favored for the prevalence of LTBI, which was primarily community-sourced (3.12 and 1.54). HCWs had an increased risk of prevalence of LTBI than the control groups, both in general hospitals and TB specific hospitals (pooled OR 2.4 and 1.57).

Conclusions: Risk of LTBI infection among HCWs is relatively high in China, especially in the eastern region, predisposed by the cumulative exposure to Mycobacterium tuberculosis from the community and the general hospitals. Overall, our data reflects an alarming risk posed to our HCWs, and calls for immediate reforms at the policy levels, so as to implement effective screening and treatment of affected HCWs in China.

Keywords: Latent tuberculosis infection (LTBI); health care workers (HCWs); prevalence; occupational diseases; China

Submitted Apr 10, 2020. Accepted for publication Jan 15, 2021.

doi: $10.21037 /$ jtd-20-1612

View this article at: http://dx.doi.org/10.21037/jtd-20-1612 


\section{Introduction}

The global burden of tuberculosis (TB) on healthcare is tremendous. TB ranks as one of the top 10 causes of mortality worldwide, even surpassing HIV/AIDS. As per the 2017 statistics, China accounted for 9\% of the global population that developed TB disease, ranking second only to India (1-3). While TB typically affects the lungs, the state of host immune response determines the symptoms of this disease. The symptoms range from a stage of clinical latency, wherein the host immune response is able to keep a check on the pathogen, to an active $\mathrm{TB}$, wherein the bacteria, Mycobacterium tuberculosis, overpowers the host immune cells (4). Latent tuberculosis infection (LTBI) is the former state that carries a dynamic balance between bacteria and the host. It is characterized by a persistent biologically positive, immune controlled stage with no clinical evidence of TB. However, the body cannot completely eliminate the pathogen from its system (5). Worldwide, nearly one-third of the global population is estimated to have LTBI (1), while in China, it ranges from $13 \%$ to $40 \%$ (6). Although only about $5-10 \%$ of LTBI cases will progress to active TB, as seen during the follow-ups (3-5), its detrimental effects on our population and healthcare systems cannot be undermined.

A study based on the longitudinal predictive model in China suggested that smear-positive patients were the main sources of new infections of TB at present, but the second related trend was the platform phase lasting for nearly 50 years. This persistence was likely to due to the remaining cases of LTBI (13-20\%), and a fairly high rate of conversion from LTBI to active TB (5-10\%) (7).

Health care workers (HCWs) are defined as people engaged in professions aimed at enhancing the wellbeing of individuals. While at work, they are commonly exposed to TB patients who may spread the infection in the hospitals even before their diagnosis is confirmed (8). It is thus, imperative to consider systematic testing and treatment of HCWs for LTBI (5). Unfortunately, LTBI testing is not widely available for HCWs (9). Until 2018, HCWs screening was often overlooked especially in lowand middle-income settings and countries $(5,10)$. The lack of data of LTBI prevalence has further aggravated the problem. The current study attempts to analyze the risk faced by HCWs in contracting LTBI, in China.

An earlier study considered two heterogeneous groups of HCWs wherein one-third (19.8 million) included administration and support workers, while the other two thirds (39.5 million) comprised of the frontline workers directly involved in providing medical treatment to the patients (9). Inconsistent conclusions among different studies were found, primarily due to the heterogeneity associated with the sampling. The risk of TB among HCWs was reported to be controversial as some studies focused on the administration groups that have no direct contacts with the TB patients, whereas others focused on the occupational groups that posed an increased risk (11). Another major factor to gauge is that whether the high risk of LTBI in HCWs is acquired from the community or the occupation (12-14). It is notable, that in low TB risk settings and countries (13) such as USA (12), Germany (14) and Iran (15), LTBI amongst HCWs primarily seems to be sourced from the community. However, the high TB risk settings and countries (16) exhibit risk derived primarily from the occupation.

Given the high probability that LTBI is likely to develop into active $\mathrm{TB}$, this meta-analysis aims to investigate the epidemiology and burden of LTBI in Chinese HCWs. Moreover, we also follow with interest the administration groups as our internal control to analyze whether the higher risk of LTBI in HCWs is community acquired or is occupational. We also surveyed if the risk remains same across different kinds of hospitals in China, i.e., general hospitals versus TB specialized hospitals.

\section{Methods}

Preferred Reporting Items for Systematic Reviews and Meta-Analyses: The PRISMA Statement was used as guideline in this meta-analysis (17).

\section{Literature search strategy}

Our search strategy seeks to identify all the accessible studies without any date or language restrictions, which reported data on the prevalence and incidence of LTBI disease among HCWs in China. We searched English databases including PubMed, Web of Science, EMBASE (Ovid), Wiley online Library, Cochrane library, the last of which was to confirm that no similar meta-analysis nor reviews have been published, so far in the given context. Chinese language articles without full text were excluded from these databases and were identified in Chinese database. Considering that a majority of studies include both TB and LTBI, we searched for studies on both subjects and then excluded the ones based exclusively on TB, in the last step. Literature published in Chinese was also obtained 
from CNKI (www.cnki.net), Wanfang (www.wanfangdata. com.cn), VIP (www.cqvip.com) and CBM database (www. sinomed.ac.cn). The Venn Diagrams of Chinese and English databases were performed in Figures S1 and S2, respectively. Search strategy has been summarized in Table S1. In addition to the above databases, we also searched for reference lists of review articles, primary studies and textbook chapters with relevant research data. We also hand searched the indices of the International fournal of Tuberculosis and Lung Disease, Tuberculosis and Tubercle \& Lung Disease for relevant articles not captured by the electronic searches. In general, keywords such as "tuberculosis", "health care workers" and "China" were used for searching the relevant articles. As an illustration, we used Medical Subject Headings (MeSH) in Pubmed database first. Secondly, the terminology "Mycobacterium tuberculosis" was substituted by the key word "tuberculosis" to enrich our search results Other terminologies such as "health care personnel", "health worker", "healthcare professional" "allied health personnel", "medical staff", "hospital staff", "physicians", "nurses", "community health worker" were substituted for "health care workers". Next, we used both the key words and subjects recognized in the databases with the operator "OR" for each terminology in each database. Finally, the keywords and subjects for each terminology were combined with an "AND" operator to obtain the final search result. This work considered data published up to September $30^{\text {th }} 2019$.

\section{Inclusion and exclusion criteria}

Literature references focusing only on the prevalence of LTBI were considered. Case-control studies, cross sectional LTBI surveys, cross-sectional cohort studies in the prevalence of LTBI among HCWs were included.

Either a tuberculin skin test (TST) or interferon-gamma release assay (IGRA) was used to confirm the presence of LTBI (5). HCWs were grouped according to the type of hospitals they were associated with, namely, the TB hospitals and the general hospitals. TB hospitals are classified as ones that have the facilities to diagnose and treat TB subjects, while general hospitals are characterized as the first to the third trinity community and country hospitals without any special TB departments. Control groups were defined as: (I) any of the administrative, finance or library staff within the studies, (II) comparable groups of non-HCWs and as per (III) the studies indicating the accurate source of the control data from national resource or elsewhere.
We excluded conference abstracts, letters and comments that did not allow extraction of relevant data, reviews as well as case reports or case series of nosocomial transmission or outbreaks. The latter is considered to be an inherently exceptional situation that may interfere in estimating the true prevalence, incidence and risk factors contributing to nosocomial transmission (13). We also excluded studies that utilized questionnaire responses to ascertain prevalence of LTBI. Inaccurate source of the control data or estimates taken from other studies, studies with ambiguous data analysis were also excluded. When the same population was studied in different papers, the most recent one or with highest quality was included.

Studies that aimed to compare LTBI in the TB associated comorbidities groups and the non-comorbidities groups were not included, primarily because comorbidities like diabetes, human immunodeficiency virus (HIV), chronic kidney disease and malignant tumor increase the risk of infection in TB populations (18). If HCWs and non-HCWs populations were later found to include these comorbidities then in that case they were not excluded.

\section{Data extraction and outcomes of interest}

These data from a subset of eligible studies were extracted independently by two reviewers (Guo and Zhong) using a standardized data extraction form. Any disagreement was resolved by the adjudicating senior authors (Wu and Qiu). Data extracted included: date of publication, author, province of study, year of study, study design, sampling method, type of HCWs, source of control group, methods that used to test for LTBI (TST or IGRA). For the prevalence and incidence studies, the following procedure was employed to standardize the data-extraction process. Data from the LTBI prevalence studies was extracted including the number of HCWs and control groups, and the associated number of positive TST or IGRA cases in HCWs and control groups, respectively. If the study conducted a series of screening methods, only the first data was considered, as subsequent screening often leads to skewness of data towards the increased risk associated with occupation. We also conducted subgroup analysis and divided China into the western, central, and eastern regions according to national geographic data (19).

\section{Quality assessment and statistical analysis}

Based on the literature database that we compiled, we investigated the study designs of only the cross-sectional 


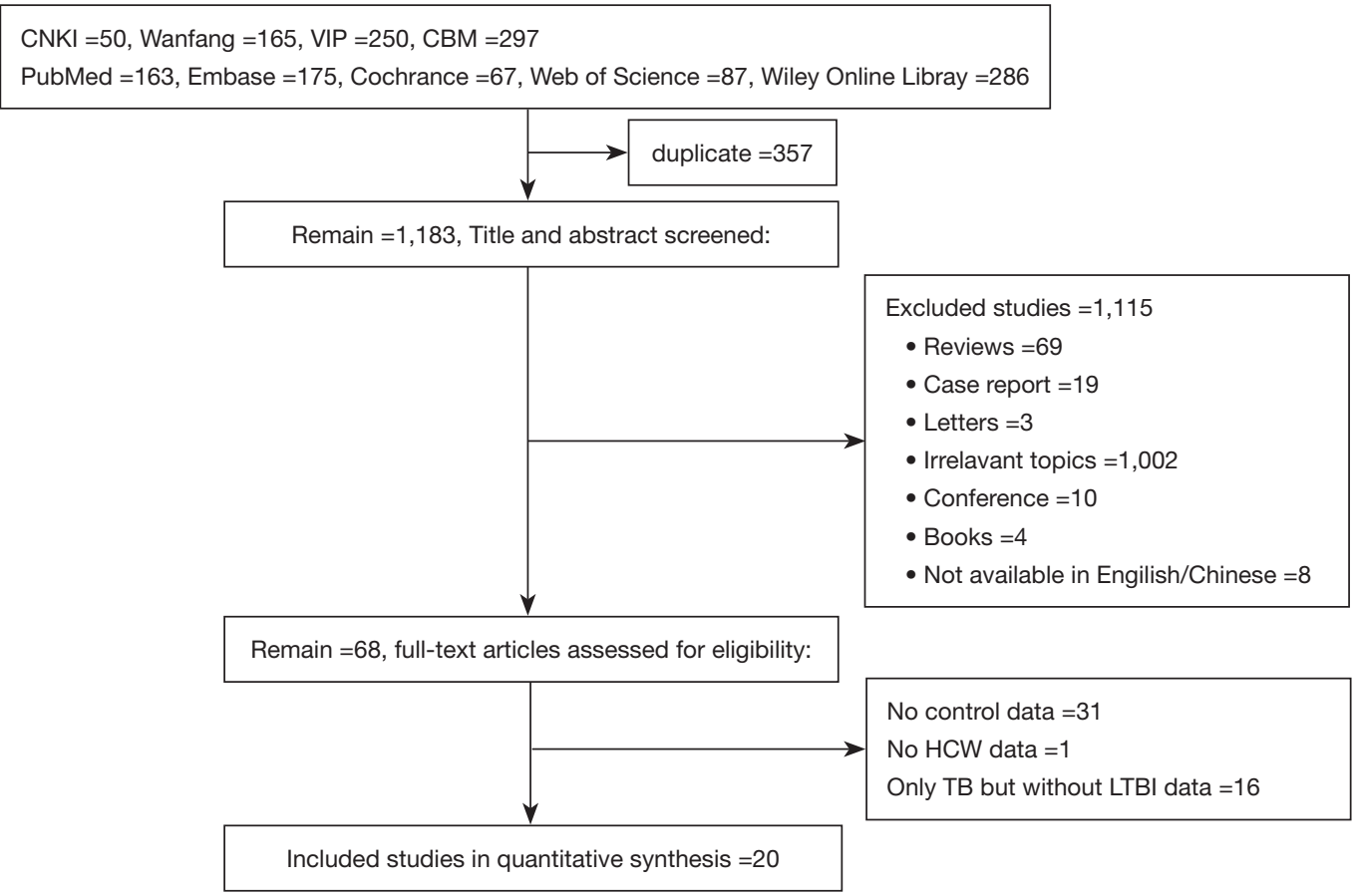

Figure 1 Flow diagram of studies identified, included, and excluded.

studies. The scale of Agency for Healthcare Research and Quality (AHRQ) provided by the US Health Care Quality And Research Institutions was used to evaluate the study quality. It contained $11 \mathrm{item} /$ parameters where in the response for each item- is either "yes", "no" or "unclear" (20). For our meta-analysis, we considered all the studies, however, during the subgroup analysis we dropped the studies that exhibited low data quality.

Review Manager 5.0 (Cochrane Collaboration, Oxford, UK) was used to perform the meta-analyses. For studies investigating the prevalence of LTBI, odds ratios (ORs) were calculated with Mantel-Haenszel (MH) method for dichotomous outcomes. All the estimates were reported with $95 \%$ confidence intervals (CIs). Chi-square test was used to assess the heterogeneity among studies, wherein a $\mathrm{P}$ value of $\mathrm{P}<0.01$ was set for significance. $\mathrm{I}^{2}$ was used to quantify statistical heterogeneity. If there was a heterogeneity between the studies, a random effect model was used, otherwise a fixed effect model was employed. Sensitivity analyses was performed for the studies that were based on the comparison of the meta-analysis results of all of the included studies and the studies that were excluded due to poor quality. Funnel plots were used to screen for potential publication bias.

Age and gender were considered to have a great influence on the relative risk observed, however, we failed to control these confounding factors as they were not accounted in most of the studies. T-test and Chi-square test was later performed to analyze whether there were age and gender differences between the HCW group and the control group, respectively. For the other confounding factors such as study period, quality of included literature, and the sampling methods, we performed subgroup analysis of the combined results, which demonstrated the reliability of our meta-analysis.

\section{Results}

\section{Study characteristics}

As shown in Figure 1, a total of 1,540 studies were identified through initial search of databases, with 8 studies in English and 12 studies in Chinese. Twenty studies were eventually included after being screened. These studies focused on the prevalence of LTBI (Table 1) and were characterized by the cross-sectional study designs. Considering that age and gender had influence on the relative risk observed, we reviewed these factors in all the included studies. Three studies provided data with stratified age and 5 studies classified data based on the gender. In the field of region 


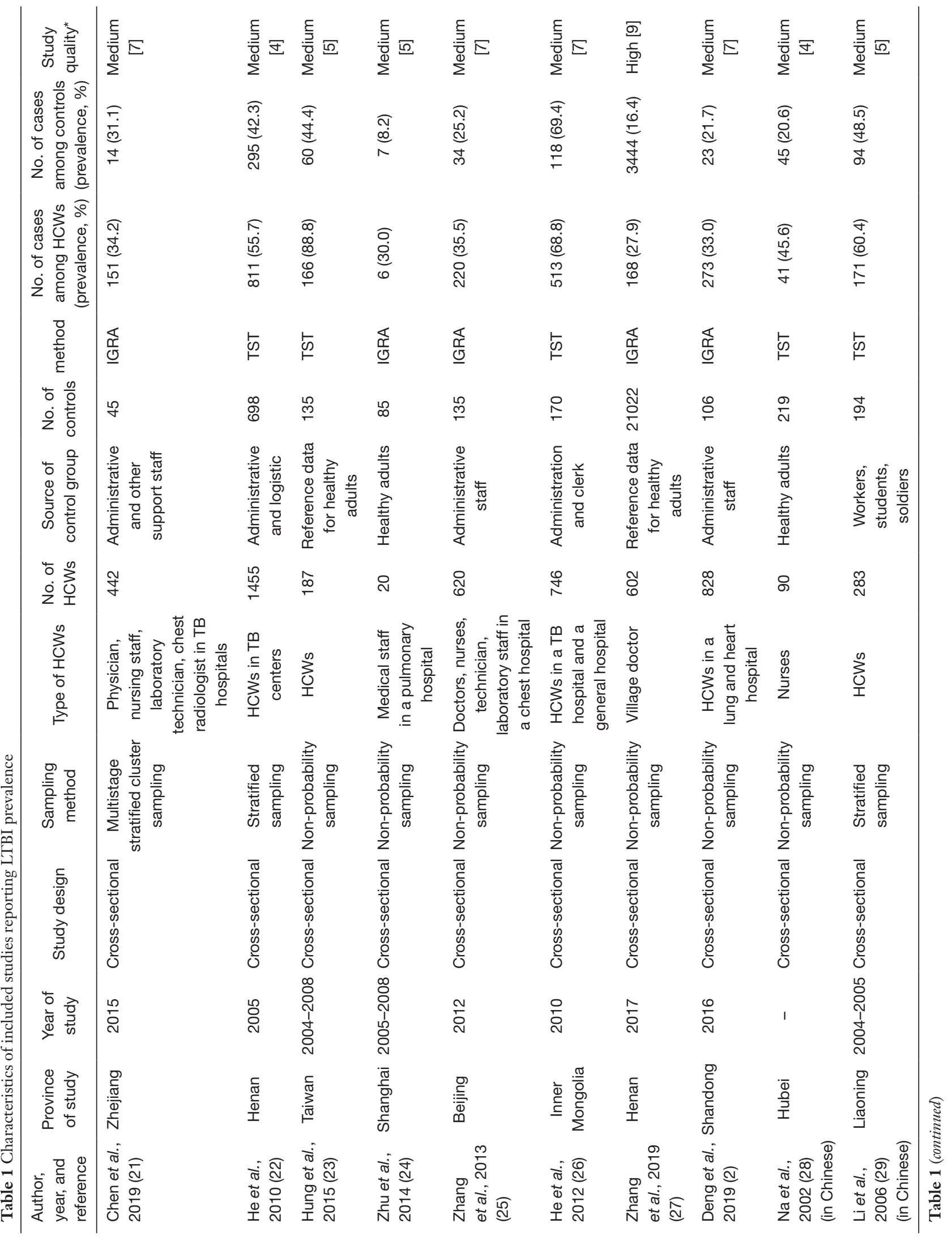




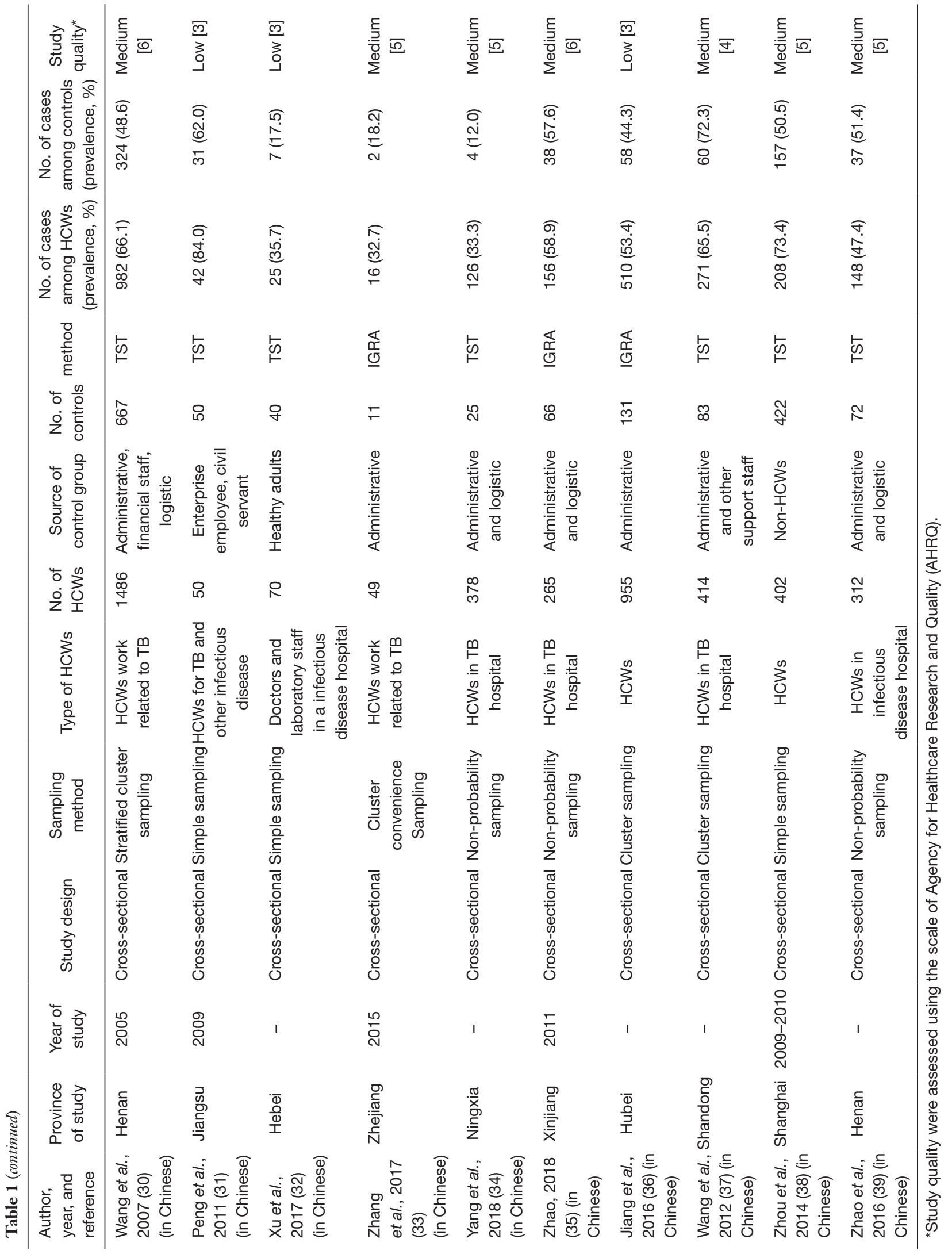


distribution, 10 studies in eastern China, 8 in central China, 2 in western China were referred. Sources of HCWs population of 13 studies came from TB (or infectious disease) specialised hospitals, 6 from general hospitals and 1 from both. The types of control groups of 12 studies were 'in-hospital' controls comprising of administrative or logistics or management personnel of the hospital. The other 6 studies considered 'community-control' where in the local population was used as control, and 1 considered both. 2 articles from the reference materials were also considered. There were 12 studies based on the diagnosis of LTBI with TST, while 8 studies with IGRA.

\section{Sensitivity}

In the 20 studies describing the prevalence of LTBI, there was a significant heterogeneity between the results after the data was combined. There was significant difference between the HCWs and the control groups $\left(\mathrm{I}^{2}=78 \%\right.$, $\mathrm{P}<0.05)$, and therefore, a random effect model was used for the combined effect analysis. Sensitivity analysis was performed later by excluding the studies one by one. This exclusion, however, did not lead to significant changes in the results, suggesting that the results of the meta-analysis were robust (Table S2).

\section{Publication bias}

Funnel plots are often used to assess publication bias in the included studies. The funnel plot results of studies describing the prevalence of LTBI appear to be asymmetric, suggesting that there may be some publication bias. There was, indeed a considerable heterogeneity between the studies. The possible reasons of this bias could be attributed to the flaws in the research design, unrigorous research methods and a small sample size, as illustrated by the wide and overlapping CIs (40). Figures have been provided to see the asymmetry (Figure S3).

In order to control the confounding factors, we performed the subgroup analysis of the combined results. First, for the subgroup analysis of the study period, it was observed that the combined OR value of the studies published before 2010 was 2.56 (95\% CI, 1.88-3.48), as compared to the OR value of 1.30 (95\% CI, 1.02-1.66) $(\mathrm{P}<0.05)$ observed for the studies published post 2010. The heterogeneity therefore, was significantly reduced with an $\mathrm{I}^{2} 83 \%$ as compared to $55 \%(\mathrm{P}<0.05)$, as seen in Figure S4. Secondly, for the subgroup analysis of the quality of the literature, it was found that the combined OR value of the studies depicting a poor quality of data was 2.06 (95\% CI, 1.49-2.84), while that with the good quality data was $1.54(95 \% \mathrm{CI}, 1.22-1.95)(\mathrm{P}<0.05)$. Accordingly, the heterogeneity was reduced slightly with the $\mathrm{I}^{2} 82 \%$ as compared to $70 \%(\mathrm{P}<0.05)$, as seen in Figure S5. Finally, for the subgroup analysis of sampling methods, the combined OR value of the studies involving non- probability sampling method was 2.01 (95\% CI, 1.37-2.96), while the studies employing probability sampling method had an OR value of 1.64 (95\% CI, 1.36-1.98) $(\mathrm{P}<0.05)$. The heterogeneity was significantly reduced, with the $\mathrm{I}^{2} 85 \%$ compared with $58 \%(\mathrm{P}<0.05)$, as seen in Figure S6. Taken together, the risk of LTBI infection among the HCWs was not significantly different and the same trends were observed after controlling the confounding factors such as the study period, the quality of included literature and the sampling method.

\section{Prevalence of LTBI}

The overall prevalence of LTBI among HCWs varied from $27.9 \%$ to $88.8 \%$, and $8.2 \%$ to $72.3 \%$ in the control groups. HCWs had a higher risk of prevalence of LTBI as compared with the control groups, with an OR of 1.78 (95\% CI, 1.46-2.16). In a cross-sectional study conducted in Taiwan, the prevalence of LTBI among HCWs was ranked the highest, reaching $88.8 \%$ (Table 1), with an OR value of 9.88 (95\% CI, 5.61-17.42; Figure 2).

\section{Consistent result for the risk of LTBI infection after considering age and gender}

As mentioned above in the method section, the age and gender based analysis for most studies were incomplete and therefore, a subgroup analysis could not be performed based on these factors. We however, performed the subgroup analysis by grouping the studies based on the presence of a local control group.

t-test was performed on 3 studies that provided the data with stratified age (Table S3). Chi-square test was performed on 5 studies that provided the data with gender grouping (Table S4). The above test results indicated whether there were age and gender differences between the HCW group and the control group. The analysis revealed that there was no statistically significant difference in the age and gender except, the one observed in the Li study ( $\mathrm{Li}$ et al., 2006). Further, subgroup analysis showed that for studies lacking 


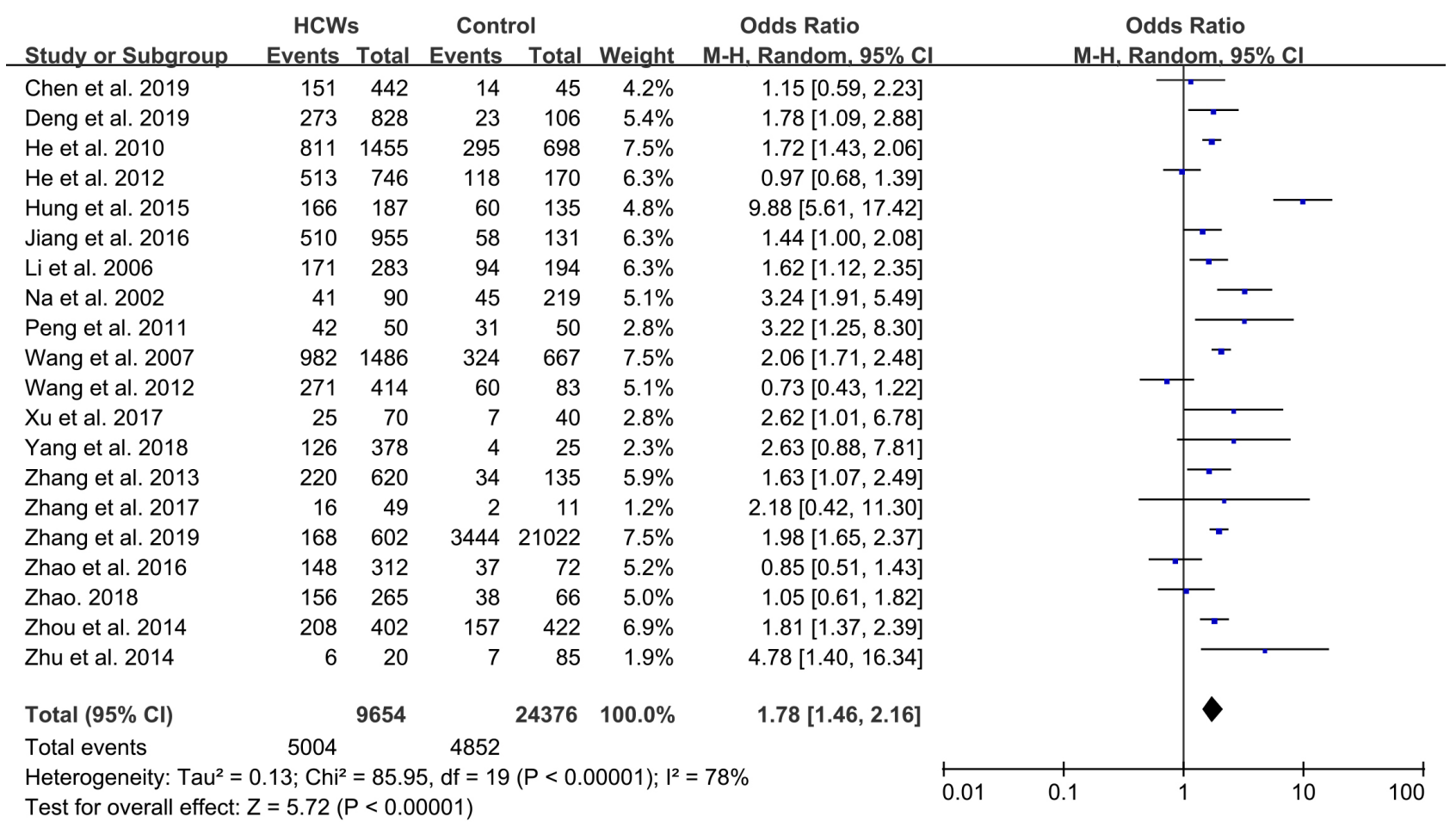

Figure 2 Forest plot showing pooled odds ratio (OR) for LTBI prevalence among HCWs. LTBI, latent tuberculosis infection; HCW, health care worker.

the local controls, the combined OR value of the risk of LTBI among HCWs was not statistically significant (OR, 4.32, 95\% CI, 0.89-21.04). In studies with local controls, and poor age and gender comparability, the heterogeneity was large $\left(\mathrm{I}^{2}=68 \%, \mathrm{P}<0.05\right)$ and therefore, a random model was used. The combined OR value of the risk of LTBI among HCWs was 1.51 (95\% CI, 1.24-1.84). In studies with local controls and good age and sex comparability, there was no heterogeneity among the studies $\left(\mathrm{I}^{2}=0, \mathrm{P}>0.05\right)$ and therefore, fixed effect model was used for analysing the data. The combined OR value of the LTBI risk among HCWs as depicted by this model was 1.94 (95\% CI, 1.50-2.51). The studies with local controls and poor age and gender comparability, and for studies with local controls and good age and gender comparability, the risk of LTBI infection among HCWs was similar and followed the same trend.

\section{Subgroup analysis of risk for prevalence of LTBI among HCWs}

In subgroup analyses of geographical location, the highest risk for the prevalence of LTBI among HCWs compared with the control group was in eastern China (OR, 2.05; 95\% CI, 1.35-3.11), followed by the central region (OR, $1.67 ; 95 \% \mathrm{CI}, 1.33-2.08)$ and the western region $(\mathrm{OR}$, 1.47; 95\% CI, 0.62-3.48) (Figure 3). Secondly, OR for LTBI compared with control groups diagnosed with TST was 1.90 (95\% CI, 1.41-2.55), while with IGRA was 1.65 (95\% CI, 1.34-2.02). Prevalence diagnosed with TST for LTBI among HCWs ranged from $33.3 \%$ to $88.8 \%$, and for control group it was observed to range from $12.0 \%$ to $72.3 \%$. When with IGRA, HCWs ranged from $27.9 \%$ to $58.9 \%$, while the control groups ranged from $8.2 \%$ to $57.6 \%$. Overall, the diagnostic positive rate of LTBI with TST was higher than with IGRA (Figure 4). Thirdly, HCWs had a higher risk for the prevalence of LTBI compared with control groups that were both communitysourced and nosocomial-sourced with a comparative higher OR for the former groups than the later groups [3.12 (95\% CI, 1.94-5.01), 1.54 (95\% CI, 1.28-1.86)] (Figure 5). Our result indicated that higher risk of LTBI in HCWs was predisposed by the community rather than nosocomial. Furthermore, HCWs in general hospitals had higher risk for the prevalence of LTBI compared with the control 


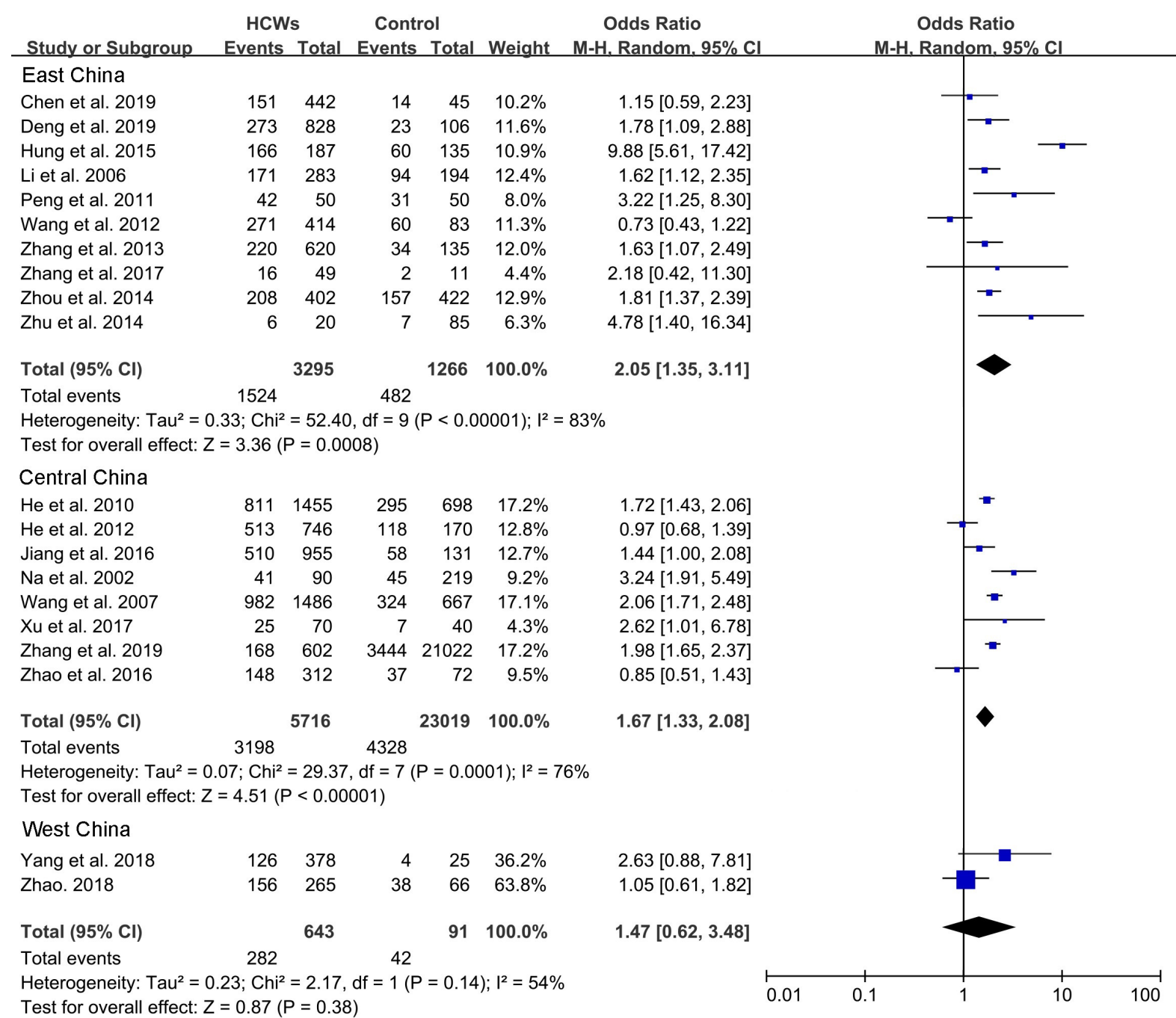

Figure 3 Forest plot showing pooled odds ratio (OR) for LTBI among HCWs according to geographic regions. LTBI, latent tuberculosis infection; HCW, health care worker.

groups (OR, 2.40; 95\% CI, 1.64-3.52). The same trend was also seen in TB or infectious disease specialist hospitals (OR, 1.57 ; 95\% CI, 1.25-1.97) (Figure 6), suggesting that the risk for the prevalence of LTBI among HCWs in general hospitals was higher than TB or infectious disease specialist hospitals.

In subgroup analysis, the control group from community wherein a general hospital and the geographical region located in the eastern China were considered, the HCWs had a higher risk of LTBI prevalence with a combined OR value ranging from 1.90-4.32. On the other hand, for the control group from a TB specialized hospital and the geographical region located in the western region, the HCWs were found to have a relatively low risk of LTBI prevalence with a combined OR value ranging from 1.47-1.65.

\section{Discussion}

Up to our knowledge, this is the first review presenting the meta-analysis of the China's nationally available data on the prevalence of LTBI among HCWs and control group, wherein the hospital's administrative staff is set as an internal control. After controlling for age and gender, we still see the same trend of the risk for LTBI infection among HCWs in China.

Our results showed an absolute risk of both LTBI and TB to HCWs than that of the general population, which was consistent with studies published earlier $(13,18,40,41)$. 


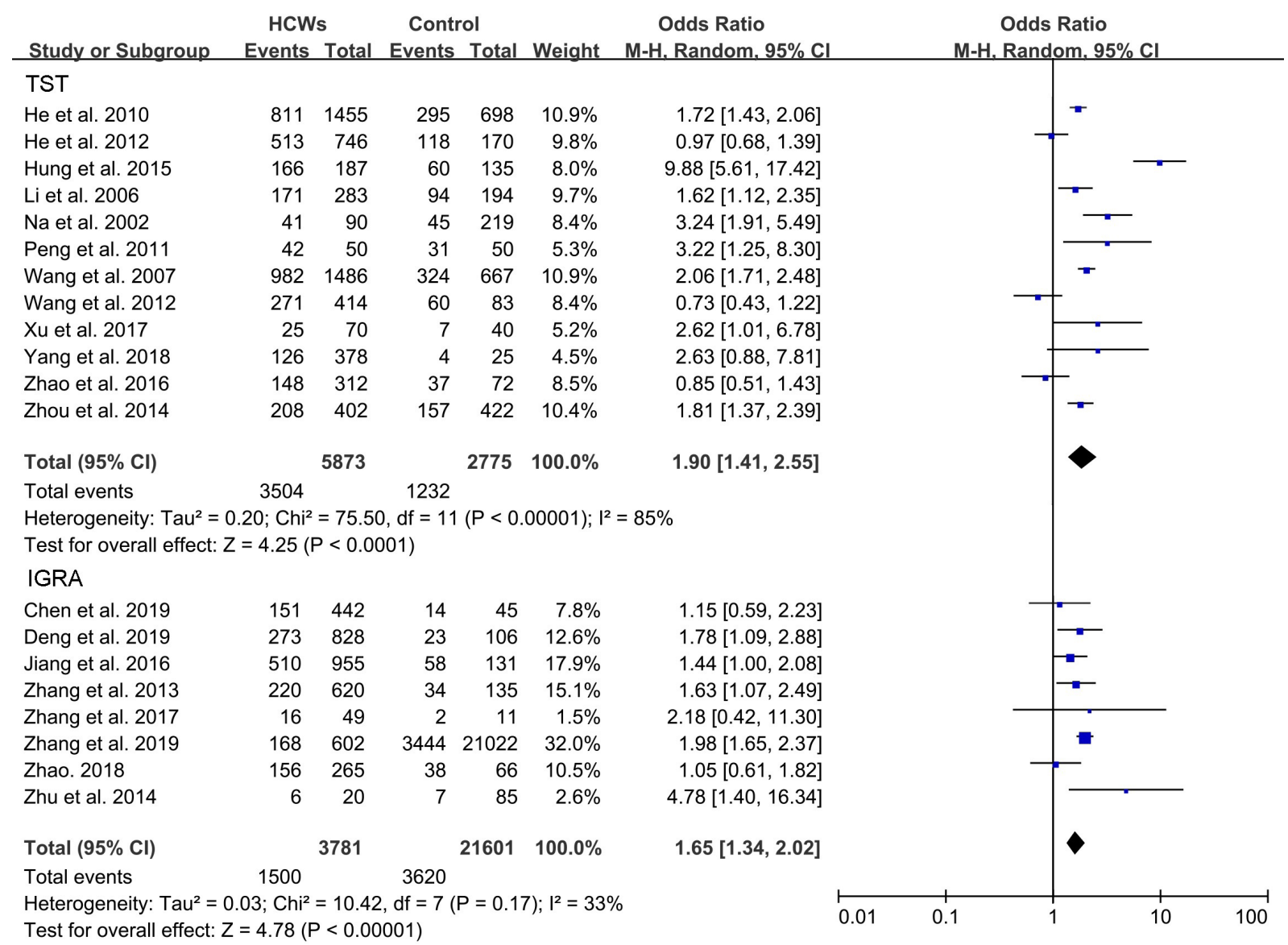

Figure 4 Forest plot showing pooled odds ratio (OR) for LTBI among HCWs according to the diagnosis methods. LTBI, latent tuberculosis infection; HCW, health care worker.

The prevalence of LTBI among HCWs was $51.5 \%$ (ranging from $27.9 \%$ to $88.8 \%$ ), which was congruous with the earlier observations made in the Low- and Middle-Income Countries (LMIC) that depicted a prevalence of $63 \%$ (range $33-79 \%$ ) (13), 54\% (range 33-79\%) (41) and 37\% (range $0.5-62.1 \%)$ (18) respectively. Since, LTBI is a reinfection or reactivation of Mycobacterium tuberculosis in the host, the former may contribute to a certain degree of immune protection, while the later predisposes to the risk of acquiring an active TB in the future (4). The results of these two interpretations with a diametrically opposite clinical outcome, may also be responsible for the large difference in the prevalence of LTBI among HCWs.

The risk of prevalence of LTBI among HCWs was found to be the highest in the eastern region of China, followed by the central and the western regions. Population density in the eastern region is particularly higher than that of the central and western regions, which leads to a higher workforce density (42). Furthermore, larger cities attract larger floating population (13) and hence, lead to higher rate of TB (13). The disparity in the economic, educational, and societal setup along with lifestyles variation in different geographic regions (43) in China resulted in HCWs in the eastern regions paying more attention to their health and thereby, resulting in effective reporting of LTBI (44). The results thus, emphasize on the importance of elevating and training HCWs for the management of $\mathrm{TB}$ as the most effective measures in preventing TB propagation. The guidelines for LTBI also recommend systematic testing and treatment of LTBI in countries with low TB incidence that may otherwise exhibit high load of LTBI prevalence (45).

The studies included in our meta-analysis involved diagnosing LTBI with TST or IGRA tests that could have led to a wide and overlapping CIs for the prevalence of LTBI. While for most studies a comprehensive description of testing protocols was provided, for a few studies it was 


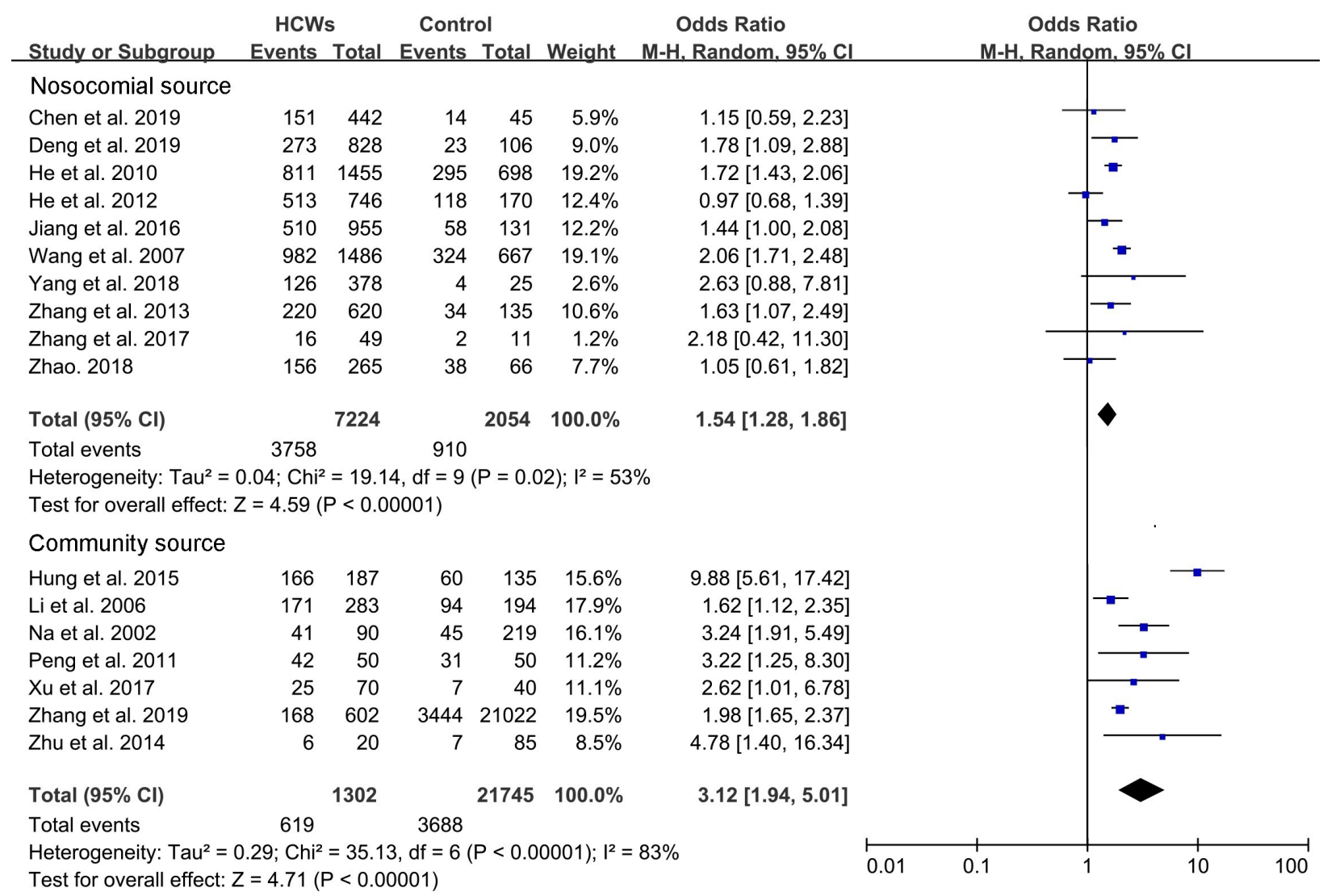

Figure 5 Forest plot showing pooled odds ratio (OR) for LTBI among HCWs according to the source of control groups. LTBI, latent tuberculosis infection; HCW, health care worker.

found to be obscure and missing important details like which kits of IGRA were used, the exact point for a positive result, whether initial two-step TST was done, how long the TST was observed, and how many units of tuberculin were used. Although, there have been arguments over the techniques best suited for effective diagnosis of LTBI; IGRA has been reported to outperform TST in settings with limited ongoing transmission and/or background prevalence of infection. The TST test, on the other hand, outperformed IGRA when the incidence and prevalence were high such as in case of an epidemic or a situation posing a high risk of infection, or when a study design warranted identification of as many infected individuals, as possible (46). It is important to note that the Bacille de Calmette Guerin (BCG)-vaccination given at birth has been reported to interfere with the results of TST, and leads to an increase in the false positive rates. IGRA however, overcomes this limitation (46). In addition to BCG, Nontuberculous Mycobacteria (NTM), occupational as well as non-occupational exposure to $M$. tuberculosis over the whole lifetime has also been reported to complicate the analysis of the prevalence of LTBI. Given the absence of any case series of nosocomial transmission or outbreaks and the fact that majority of the Chinese population is BCGvaccinated, IGRA outperformed TST in detecting the prevalence of LTBI among the HCWs. Another important difference reported in literature shows that between IGRA and TST, IGRA is more likely to detect recent infection of TB, whereas TST detects the cumulative exposure to M. tuberculosis over time $(47,48)$. Together, our findings highlight that the risk of prevalence of LTBI diagnosed with TST was higher than IGRA and hence, it can be speculated that Chinese HCWs had more cumulative exposure to TB than recent infections. Although, neither TST nor IGRA are gold standards for the diagnosis of LTBI, they are relevant in detecting the host immune response. It is thus, recommended to undertake further combination tests or gene testing techniques to reach to a more accurate and 


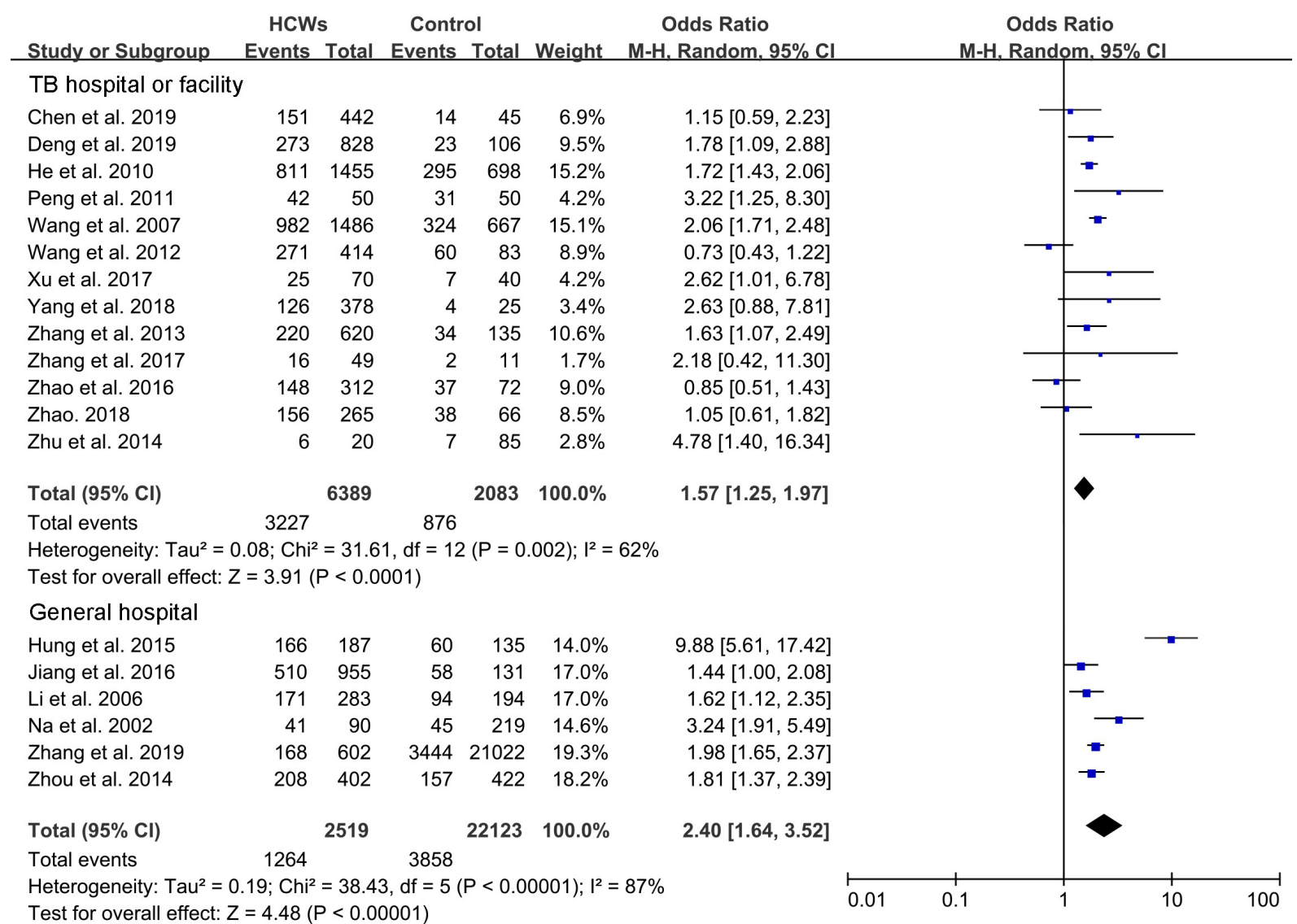

Figure 6 Forest plot showing pooled odds ratio (OR) for LTBI among HCWs according to the type of hospitals. LTBI, latent tuberculosis infection; HCW, health care worker.

conclusive analysis.

To the best of our knowledge, we are the first to report that the risk of prevalence of LTBI among Chinese HCWs population is largely sourced from the community as against the nosocomial infection. Our results differ from the previous findings that reflected that LTBI among HCWs is primarily derived through nosocomial infections (13). These earlier studies however, lacked comparable or contemporaneous controls and only relied on national data for analysis. Moreover, no studies from China were included in their analysis (13). Uden and his Colleagues did provide a comparable and contemporaneous control data as well as, research data from China, but it did not provide evidence for the source of infection, whether nosocomial or community-derived (18). We suggest that further molecular research studies involving DNA fingerprinting, rather than TST or IGRA, must be undertaken to provide verifying evidence of nosocomial contagion (41).

Among the different type of hospitals that we studied, we observed that HCWs in general hospitals showed a higher risk of prevalence of LTBI. This trend was consistent with that in the subgroup analysis of community and nosocomial source, as discussed above. This could be partly because the risk of prevalence of LTBI among HCWs in general hospital is primarily community-sourced.

There are still some limitations in our meta-analysis. First, our study did not analyze age stratification and gender comparability. However, we compared the studies with local controls and poor age and gender comparability, and the studies with local controls and good age and gender comparability. Our analysis showed that the risk of LTBI infection among HCWs remains the same and follows the same trend. This might reflect a more complex aspect of the problem. Secondly, it is notable that a systemic review has its own limitation of publication bias, multiple publication bias and the possibility to miss out the studies being researched. In our analysis, we observed a considerable heterogeneity between the studies, reflecting 
different settings and populations. HCWs have always been concerned about the risk of developing TB, which might have remained under reported due to heterogenic composition of professionals involved in healthcare services. However, the majority of studies included in our dataset had a clear inclusion criterion for HCWs and control groups that reduced the selection bias. Finally, publications only in English and Chinese were included, which may result in language bias. However, only Chinese HCWs were the main focus of our study where the relevant published data/ papers are in English and Chinese language in majority.

In the end, it is true that the precise prevalence of LTBI in the Chinese HCWs population is not accurate and is also difficult to estimate precisely. We attempt to ignite Chinese scholars to take an interest in this real and complex situation about LTBI in China. The observed complexity is a result of pitfalls in the methodologies. For example: a positive TST can be a false positive if not followed by an IGRA due to a BCG vaccination or to a previous TST. The absence of clear distinctions based on age and gender also results in lacking of another important information-the elderly are expected to be more vulnerable to LTBI. Furthermore, the presence of internal controls within a hospital may not be considered as ideal comparison due to the airborne infection risk, even they are not directly dealing with the patients. All the above observations should be considered for developing a better understanding of the spread of infection amongst the working populations exposed to the risk in China.

\section{Conclusions}

We provide a national epidemiological basis for the development of LTBI management in HCWs in China. Our study shows that that risk of LTBI infection among HCWs was relatively high in China, especially in the eastern region. Furthermore, we observe that the primary source of LTBI is cumulative exposure to Mycobacterium TB infection, derived from the community. Compared to TB specialized hospitals the associated risk was found to be higher in the HCWs of the general hospitals. Overall, our result emphasizes on a pressing need to strengthen the national policies in China so as to curb the spread of LTBI among HCWs. In order to protect the HCWs, the hospitals need to adopt and implement the following approaches: (I) education services need to be provided for the occupational risks associated with LTBI; (II) personal protective equipments must be provide to prevent contraction of infection; (III) effective screening and treatment should be supplied. In addition, the methodology and the subsequent limitations presented in current study might serve as instrumental approaches for more comprehensive and accurate study designs involving gender, age, type of hospitals and geography, which may help improve estimating accuracy of the LTBI prevalence in HCWs. Thus, our study provides strong basis for the formulation of strategies to effectively curb the high prevalence of LTB1 in HCWs as well as other individuals in China.

\section{Acknowledgments}

Funding: This project was supported by A Major Infectious Disease Prevention and Control of the National Science and Technique Major Project (2017ZX10201302005002, 2018ZX10715004-002-001) and The Medical Research Fund Project of Guangdong Province (B2020013).

\section{Footnote}

Conflicts of Interest: All authors have completed the ICMJE uniform disclosure form (available at http://dx.doi. org/10.21037/jtd-20-1612). All authors report grants from A Major Infectious Disease Prevention and Control of the National Science and Technique Major Project (No. 2017ZX10201302005002), grants from A Major Infectious Disease Prevention and Control of the National Science and Technique Major Project (No. 2018ZX10715004-002), non-financial support from The Medical Research Fund Project of Guangdong Province (No. B2020013), during the conduct of the study.

Ethical Statement: The authors are accountable for all aspects of the work in ensuring that questions related to the accuracy or integrity of any part of the work are appropriately investigated and resolved.

Open Access Statement: This is an Open Access article distributed in accordance with the Creative Commons Attribution-NonCommercial-NoDerivs 4.0 International License (CC BY-NC-ND 4.0), which permits the noncommercial replication and distribution of the article with the strict proviso that no changes or edits are made and the original work is properly cited (including links to both the formal publication through the relevant DOI and the license). See: https://creativecommons.org/licenses/by-nc-nd/4.0/.

\section{References}


1. World Health O. Global tuberculosis report 2018. Geneva: World Health Organization; 2018.

2. Deng Y, Liu Y, Li Y, et al. Isolation measures and protection awareness are significant for latent tuberculosis infection: a cross-sectional study based on T-SPOT.TB among health care workers in China. Epidemiol Infect 2019; 147:e120.

3. Targeted tuberculin testing and treatment of latent tuberculosis infection. This official statement of the American Thoracic Society was adopted by the ATS Board of Directors, July 1999. This is a Joint Statement of the American Thoracic Society (ATS) and the Centers for Disease Control and Prevention (CDC). This statement was endorsed by the Council of the Infectious Diseases Society of America. (IDSA), September 1999, and the sections of this statement. Am J Respir Crit Care Med 2000;161:S221-47.

4. Pai M, Denkinger CM, Kik SV, et al. Gamma interferon release assays for detection of Mycobacterium tuberculosis infection. Clin Microbiol Rev 2014;27:3-20.

5. World Health O. Latent tuberculosis infection: updated and consolidated guidelines for programmatic management: annex 3: values and preferences for the management of latent tuberculosis infection: survey of populations affected by the recommendations. Geneva: World Health Organization2018 2018 Contract No.: WHO/CDS/TB/2018.9.

6. Gao L, Lu W, Bai L, et al. Latent tuberculosis infection in rural China: baseline results of a population-based, multicentre, prospective cohort study. The Lancet Infect Dis 2015;15:310-9.

7. Xu K, Ding C, Mangan CJ, et al. Tuberculosis in China: A longitudinal predictive model of the general population and recommendations for achieving WHO goals. Respirology 2017;22:1423-9.

8. Hung HF, Wang CW, Hsu CH, et al. Sharing experiences of abnormal admissions to a regional hospital for patients with tuberculosis. Int J Antimicrob Agents 2017;50:S225-S.

9. Magowe M. Joint WHO/ILO policy guidelines on improving health worker access to prevention, treatment and care services for HIV and TB. 2010.

10. World Health O. Use of tuberculosis interferon-gamma release assays (IGRAs) in low- and middle-income countries: policy statement. Geneva: World Health Organization; 2011.

11. Seidler A, Nienhaus A, Diel R. Review of epidemiological studies on the occupational risk of tuberculosis in low- incidence areas. Respiration 2005;72:431-46.

12. Larsen NM, Biddle CL, Sotir MJ, et al. Risk of tuberculin skin test conversion among health care workers: occupational versus community exposure and infection. Clin Infect Dis 2002;35:796-801.

13. Menzies D, Joshi R, Pai M. Risk of tuberculosis infection and disease associated with work in health care settings. Int J Tuberc Lung Dis 2007;11:593-605.

14. Schablon A, Beckmann G, Harling M, et al. Prevalence of latent tuberculosis infection among health care workers in a hospital for pulmonary diseases. J Occup Med Toxicol 2009;4:1.

15. Salmanzadeh S, Abbasissifar H, Alavi SM. Comparison study of QuantiFERON test with tuberculin skin testing to diagnose latent tuberculosis infection among nurses working in teaching hospitals of Ahvaz, Iran. Caspian J Intern Med 2016;7:82-7.

16. Nienhaus A, Schablon A, Preisser AM, et al. Tuberculosis in healthcare workers - a narrative review from a German perspective. J Occup Med Toxicol 2014;9:9.

17. Moher D, Liberati A, Tetzlaff J, et al. Preferred reporting items for systematic reviews and meta-analyses: the PRISMA statement. PLoS Med 2009;6:e1000097.

18. Uden L, Barber E, Ford N, et al. Risk of Tuberculosis Infection and Disease for Health Care Workers: An Updated Meta-Analysis. Open Forum Infect Dis 2017;4:ofx137.

19. Fan CC. Uneven development and beyond: regional development theory in post-Mao China. 1997;21:620-39.

20. Owens DK, Lohr KN, Atkins D, et al. AHRQ series paper 5: grading the strength of a body of evidence when comparing medical interventions--agency for healthcare research and quality and the effective health-care program. J Clin Epidemiol 2010;63:513-23.

21. Chen B, Gu H, Wang X, et al. Prevalence and determinants of latent tuberculosis infection among frontline tuberculosis healthcare workers in southeastern China: A multilevel analysis by individuals and health facilities. Int $\mathrm{J}$ Infect Dis 2019;79:26-33.

22. He GX, van den Hof S, van der Werf MJ, et al. Infection control and the burden of tuberculosis infection and disease in health care workers in china: a cross-sectional study. BMC Infect Dis 2010;10:313.

23. Hung WT, Lee SS, Sy CL, et al. Prevalence of latent tuberculosis infection in BCG-vaccinated healthcare workers by using an interferon-gamma release assay and the tuberculin skin test in an intermediate tuberculosis burden country. J Microbiol Immunol Infect 2015;48:147-52. 
24. Zhu C, Liu Z, Li Z, et al. The performance and limitation of T-SPOT.TB for the diagnosis of TB in a high prevalence setting. J Thorac Dis 2014;6:713-9.

25. Zhang X, Jia H, Liu F, et al. Prevalence and Risk Factors for Latent Tuberculosis Infection among Health Care Workers in China: A Cross-Sectional Study. Plos One 2013;8:e66412.

26. He GX, Wang LX, Chai SJ, et al. Risk factors associated with tuberculosis infection among health care workers in Inner Mongolia, China. Int J Tuberc Lung Dis 2012;16:1485-91.

27. Zhang H, Xin H, Wang D, et al. Serial testing of Mycobacterium tuberculosis infection in Chinese village doctors by QuantiFERON-TB Gold Plus, QuantiFERON-TB Gold in-Tube and T-SPOT.TB. J Infect 2019;78:305-10.

28. Na ML, Wang YG, Li XZ. Investigation of tuberculosis infection in clinical nurses. Chin J Nosocomiol. Chin J Nosocomiol 2002;12:758-9.

29. Li JM, Lu W, W DL, et al. Discussion on the risk of tuberculosis infection among medical staff. Chin J Nosocomiol 2006;07:745-7.

30. Wang GJ, Ma SW, Yan XA, et al. Investigation on tuberculosis infection rate of workers in tuberculosis control institutions in Henan Province. Chin J Epidemiol 2007;28:980-3.

31. Peng WL, Liu CY, Zhang HQ, et al. The status of tuberculosis latent infection and analysis to laboratory monitoring indicators in high-risk medical staff. J Clin Pulm Med 2011;16:546-7.

32. Xu Y, Wang YL, Yan NX, et al. Analysis of laboratory monitoring indexes of latent tuberculosis infection in highrisk medical staff. J Clin Pulm Med 2017;22:41-3+53.

33. Zhang SC, Wang WH, Zhong JF, et al. Survey on the tuberculosis infection among health care workers for tuberculosis in Huzhou City. Chin Prev Med 2017;18:902-5.

34. Yang ZG, Ma LL, Cheng RX. Analysis of tuberculosis infection status and risk factors among staff in designated hospitals in Ningxia. Anhui J Prev Med 2018;24:301-4.

35. Zhao B. Dynamic analysis of mycobacterium tuberculosis infection in medical personnel: Chinese Center for Disease Control and Prevention; 2018.

36. Jiang J, Zhang W. Investigation and analysis on risk factors of mycobacterium tuberculosis infection among the medical staff in general hospital. Chin J Exp Clin Infect Dis 2016;10:712-5.

37. Wang FT, Deng YF, Li Y, et al. Study on the risk of tuberculosis infection among medical staff in tuberculosis hospitals. Chin J Nosocomiol 2012;22:1674-6.

38. Zhou Y. Clinical significance of tuberculin purified protein derivative in test of mycobacterium tuberculosis infection in hospital. Chin J Nosocomiol 2014;24:4418-20.

39. Zhao XL, Li XL, Zhou F, et al. Survey on tuberculosis infection among health care workers in an infectious diseases hospital. Chin J Infect Control 2016;15:93-6.

40. Nasreen S, Shokoohi M, Malvankar-Mehta MS. Prevalence of Latent Tuberculosis among Health Care Workers in High Burden Countries: A Systematic Review and Meta-Analysis. PLoS One 2016;11:e0164034.

41. Joshi R, Reingold AL, Menzies D, et al. Tuberculosis among health-care workers in low- and middle-income countries: a systematic review. PLoS Med 2006;3:e494.

42. Shi Y, Xu B, Wang WB, et al. Equity study in health workforce on tuberculosis control in China: a nationwide evaluation. Biomed Environ Sci 2013;26:702-7.

43. National Bureau of Statistics of China, the sixth national census reports. 2010.

44. Li CY, Sung FC. A review of the healthy worker effect in occupational epidemiology. Occup Med (Lond) 1999;49:225-9.

45. Disease GBD, Injury I, Prevalence C. Global, regional, and national incidence, prevalence, and years lived with disability for 354 diseases and injuries for 195 countries and territories, 1990-2017: a systematic analysis for the Global Burden of Disease Study 2017. Lancet (London, England) 2018;392:1789-858.

46. Leung CC, Yam WC, Yew WW, et al. T-Spot.TB outperforms tuberculin skin test in predicting tuberculosis disease. Am J Respir Crit Care Med 2010;182:834-40.

47. Adams S, Ehrlich R, Baatjies R, et al. Predictors of discordant latent tuberculosis infection test results amongst South African health care workers. BMC Infect Dis 2019;19:131.

48. Dorman SE, Belknap R, Graviss EA, et al. Interferongamma release assays and tuberculin skin testing for diagnosis of latent tuberculosis infection in healthcare workers in the United States. Am J Respir Crit Care Med 2014;189:77-87.

Cite this article as: Guo HY, Zhong QH, Zhou J, Zhao ZM, Zhang XL, Chen ZH, Qiu XC, Wu ZL. Risk of prevalence of latent tuberculosis infection in health care workers-an idiographic meta-analysis from a Chinese perspective. J Thorac Dis 2021;13(4):2378-2392. doi: 10.21037/jtd-20-1612 


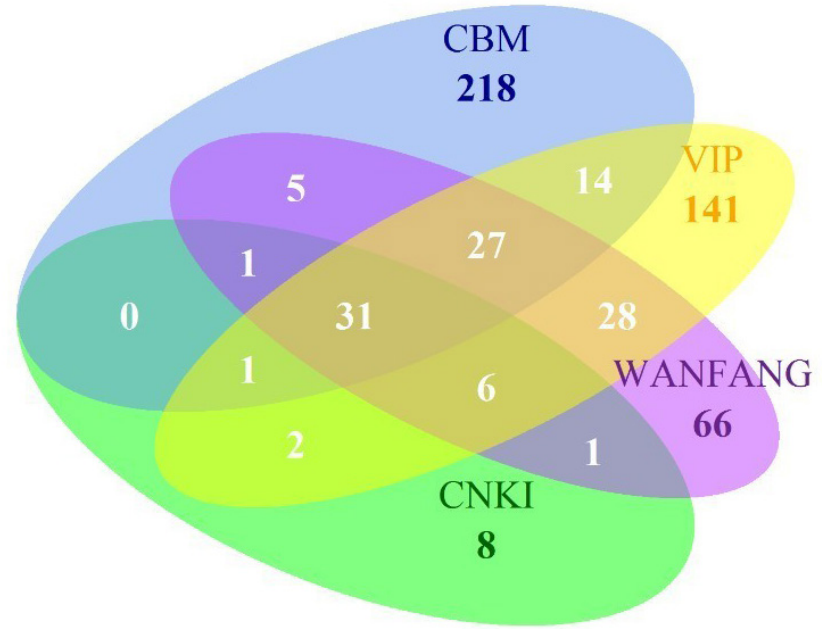

Figure S1 Venn Diagram of Chinese Databases. There were four Chinese databases including the CBM, VIP, WANFANG, and CNKI, which had a total of 762 articles. These were 297 articles in the CBM, 250 articles in the VIP, 165 articles in the Wanfang and 50 articles in the CNKI. Articles without duplicates among databases included 218 in the CBM, 141 in the VIP, 66 in the Wanfang and 8 in the CNKI. There were 14 duplicate articles between the CBM and the VIP databases, 5 duplicate articles between the CBM and the WANFANG databases, 28 duplicate articles between the VIP and the WANFANG databases, 2 duplicate articles between the VIP and the CNKI databases, 1 duplicate article between the WANFANG and the CNKI databases, and no article were found duplicated in the CBM and the CNKI databases. In addition, there were 27 duplicate articles through the CBM, VIP and WANFANG databases, 1 duplicate article through the CBM, VIP and CNKI databases, 1 duplicate article through the CBM, WANFANG and CNKI databases, 6 duplicate articles through the VIP, WANFANG and CNKI databases. Furthermore, 31 duplicate articles were found across four databases. 


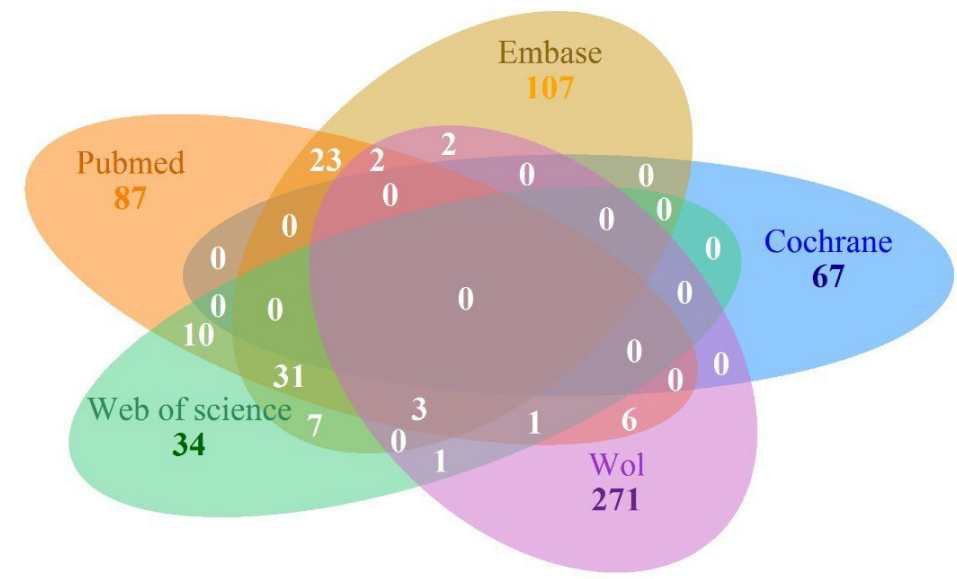

Figure S2 Venn Diagram of English Databases. There were five English databases including the Embase, Pubmed, Web of science, Wiley online library (Wol) and Cochrane, which had a total of 778 articles. These were 175 articles in the Embase, 163 articles in the Pubmed, 87 articles in the Web of science, 286 articles in the Wol and 67 articles in the Cochrane. Articles without duplicates among databases included 67 in the Cochrane, 107 in the Embase, 87 in the Pubmed, 34 in the Web of science and 271 in the Wol. There was no duplicate article between the Cochrane and other databases. In addition, there were 23 duplicate articles between the Embase and the Pubmed databases, 7 duplicate articles between the Embase and the Web of science databases, 2 duplicate articles between the Embase and the Wol databases, 10 duplicate articles between the Pubmed and the Web of science databases, 6 duplicate articles between the Pubmed and the Wol databases, 1 duplicate article between the Web of science and the Wol databases. Furthermore, there were 31 duplicate articles through the Embase, Pubmed and Web of science databases, 2 duplicate articles through the Embase, Pubmed and Wol databases, 1 duplicate article through the Pubmed, Web of science and Wol databases, and no duplicate articles through the Embase, Web of science and Wol databases. Finally, 3 duplicate articles were found across the Embase, Pubmed, Web of science and Wol databases. 
Table S1 Details of database, journals and grey literature search

\begin{tabular}{ll}
\hline Database & \multicolumn{1}{c}{ Search words } \\
\hline Pubmed & $(((((($ tuberculosis) OR mycobacterium tuberculosis) OR TB)) AND $(((()((()((()((()((()(($ "health personnel") OR " health care personnel") OR "healthcare \\
& personnel") OR "health care worker") OR " health care workers") OR "healthcare worker") OR "healthcare workers") OR "health worker") \\
& OR "health workers") OR "health professional") OR "health professionals") OR "health care professional") OR "health care professionals") \\
& OR "healthcare professional") OR "healthcare professionals") OR "medical care personnel") OR "nurse") OR "nurses") OR "nursing") OR \\
& physician) OR physicians) OR HCW)) OR (((((((")allied health personnel") OR caregivers) OR "medical staff") OR "nurses") OR "nursing staff") \\
& OR personnel,hospital) OR physicians))) AND (((((("Infection Control") OR "Cross Infection") OR "Occupational Diseases") OR prevalence) \\
& OR incidence) OR (incidence[Title/Abstract] OR prevalence[Title/Abstract])) AND (((Macao[MeSH Terms]) OR (((China[MeSH Terms]) OR \\
& hongkong[MeSH Terms]) OR taiwan[MeSH Terms])))
\end{tabular}

Web of science TS=tuberculosis OR TS="mycobacterium tuberculosis" OR TS=TB

Date of search

Number of records

TS=tuberculosis OR TS="mycobacterium tuberculosis" OR TS=TB

30 September

163

2019

OR TS="healthcare worker" OR TS="healthcare workers" OR TS="health worker" OR TS="health workers" OR TS="health professional"

OR TS="health professionals" OR TS="health care professional" OR TS="health care professionals" OR TS="healthcare professional" OR

TS="healthcare professionals" OR TS="medical care personnel" OR TS="nurse" OR TS="nurses" OR TS="nursing" OR TS=physician OR

TS=physicians OR TS=HCW OR TS="allied health personnel" OR TS=caregivers OR TS="medical staff" OR TS="nurses" OR TS="nursing staff"

OR TS="personnel,hospital" OR TS=physicians

TS="Infection Control" OR TS="Cross Infection" OR TS="Occupational Diseases" OR TS=prevalence OR TS=incidence OR Tl=incidence OR

$\mathrm{TI}=$ prevalence

TS=Macao OR TS=China OR TS=hongkong OR TS=Taiwan

1 AND 2 AND 3 AND 4

Embase

1."tuberculosis"/exp or "tuberculosis".mp. or "mycobacterium tuberculosis"/exp or "mycobacterium tuberculosis".mp. or "TB"/exp or "TB".mp. 2.("health personnel" or " health care personnel" or "healthcare personnel" or "health care worker" or "health care workers" or "healthcare worker" or "healthcare workers" or "health worker" or "health workers" or "health professional" or "health professionals" or "health care professional" or "health care professionals" or "healthcare professional" or "healthcare professionals" or "medical care personnel" or "nurse" or "nurses" or "nursing" or physician or physicians or HCW or "allied health personnel" or caregivers or "medical staff" or "nursing staff" or personnel,hospital or physicians).mp.

3."Infection Control"/exp or "Infection Control".mp. or "Cross Infection"/exp or "Cross Infection".mp. or "Occupational Diseases"/exp or "Occupational Diseases".mp. or prevalence/exp or prevalence.mp. or incidence/exp or incidence.mp.

4.Macao/exp or Macao.mp. or China/exp or China.mp. or hongkong/exp or hongkong.mp. or Taiwan/exp or Taiwan.mp. 1 AND 2 AND 3 AND 4

Cochrane (tuberculosis) OR mycobacterium tuberculosis) OR TB OR "latent tuberculosis" OR "mycobacterium tuberculosis" in All Text AND "health personnel" OR " health care personnel" OR "healthcare personnel" OR "health care worker" OR "health care workers" OR "healthcare worker" OR "healthcare workers" OR "health worker" OR "health workers" OR "health professional" OR "health professionals" OR "health care professional" OR "health care professionals" OR "healthcare professional" OR "healthcare professionals" OR "medical care personnel" OR "nurse" OR "nurses" OR "nursing" OR physician OR physicians OR HCW OR "allied health personnel" OR caregivers OR "medical staff" OR "nursing staff" OR (personnel,hospital) OR physicians in All Text AND "Infection Control" OR "Cross Infection" OR "Occupational Diseases" OR prevalence OR incidence in All Text AND Macao OR China OR hongkong OR Taiwan in All Text - (Word variations have been searched)

Wiley online library "((tuberculosis) OR mycobacterium tuberculosis) OR TB OR "latent tuberculosis" OR "mycobacterium tuberculosis"" in Abstract and" "hea Ith personnel" OR " health care personnel" OR "healthcare personnel" OR "health care worker" OR "health care workers" OR "healthcare worker" OR "healthcare workers" OR "health worker" OR "health workers" OR "health professional" OR "health professionals" OR "health care professional" OR "health care professionals" OR "healthcare professional" OR "healthcare professionals" OR "medical care personnel" OR "nurse" OR "nurses" OR "nursing" OR physician OR physicians OR HCW OR "allied health personnel" OR caregivers OR "medical staff" OR "nursing staff" OR (personnel,hospital) OR physicians" anywhere and " "Infection Control" OR "Cross Infection" OR "Occupational Diseases" OR prevalence OR incidence" anywhere and "Macao OR China OR hongkong OR Taiwan" in Abstract

CNKI (Chinese) Tl=('tuberculosis' + 'tuberculosis mycobacteria' + 'pulmonary tuberculosis') * (('hospital' * ('staff' + 'worker')) + 'medical institution' + 'medical personnel' + 'doctor' + 'Nurse' + 'Medical staff' + 'Nursing staff' + 'Physician' + 'Medical worker' + 'Tuberculosis control staff') * ('Cross infection' + 'Medical infection' + 'Occupational disease' + 'Potential Infection ' + 'latent infection' + 'infection' + 'epidemic' + 'incidence' + 'prevalence')

Wanfang(Chinese) Title or keyword:(“tuberculosis" + "tuberculosis mycobacteria" + "pulmonary tuberculosis") * (“"hospital” * ("staff" + "worker")) + "medical institution" + "medical personnel" + "doctor" + "Nurse" + "Medical staff" + "Nursing staff" + "Physician" + "Medical worker" + "Tuberculosis control staff") * ("Cross infection" + "Medical infection" + "Occupational disease" + "Potential Infection" + "latent infection" + "infection" + "epidemic" + "incidence" + "prevalence")

VIP(Chinese) $\quad \mathrm{M}=$ (tuberculosis OR tuberculosis mycobacteria OR pulmonary tuberculosis) AND ((hospital AND (staff OR worker)) OR medical institution OR medical personnel OR doctor OR Nurse OR Medical staff OR Nursing staff OR Physician OR Medical worker OR Tuberculosis control staff) AND (Cross infection OR Medical infection OR Occupational disease OR Potential Infection OR latent infection OR infection OR epidemic OR incidence OR prevalence)

CBM(Chinese) (“tuberculosis"[title] + "tuberculosis mycobacteria"[title] + "pulmonary tuberculosis"'[title]) * ((“hospital”[title] * ("staff”[title] + "worker”[title])) + "medical institution"[title] + "medical personnel"[title] + "medical personnel"[Common field] + "doctor"[title] + "Nurse"[title] + "Medical staff"[ + "Nursing staff" [title] + "Physician"[title] + "Medical worker"[title] + "Tuberculosis control staff"[title]) * ("Cross infection"[title] +Health carerelated infections [[Common fields] + "Health-related infections" [Common fields] + "Medical-related infections" [Common fields] + "Hospita infections" [Common fields] + "Medical infection"[title] + "Occupational disease"[title] + "Potential Infection"[title] + "latent infection"[title] + "infection"[title] + "epidemic"[title] + "incidence"[title] + "prevalence"[title]) 
Table S2 Sensitivity analysis after excluding studies one by one reporting LTBI prevalence

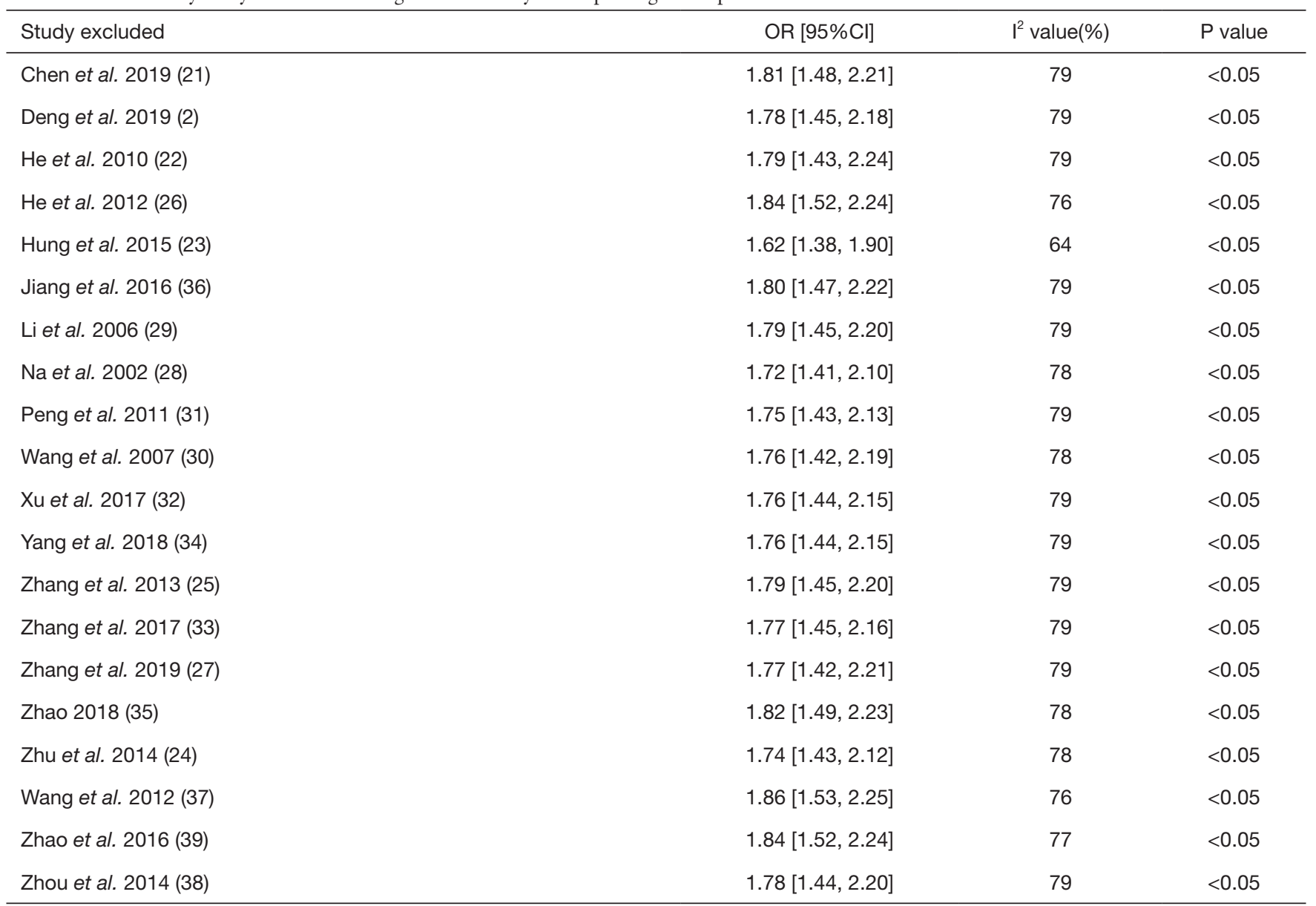




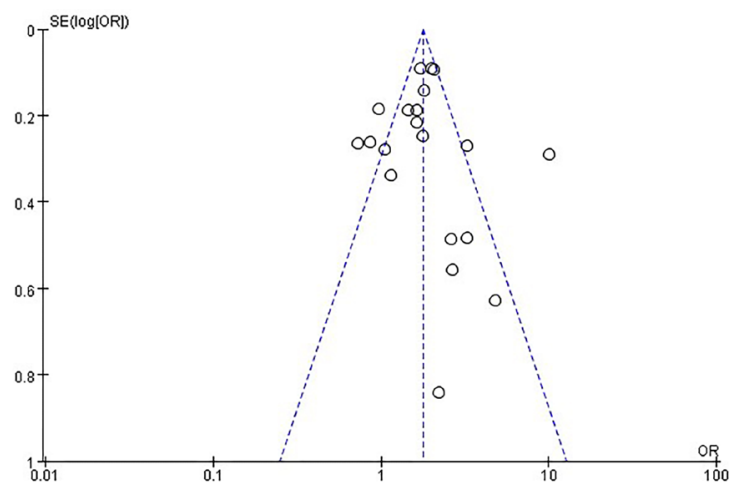

Figure S3 Funnel plot of included studies on LTBI prevalence among HCWs.

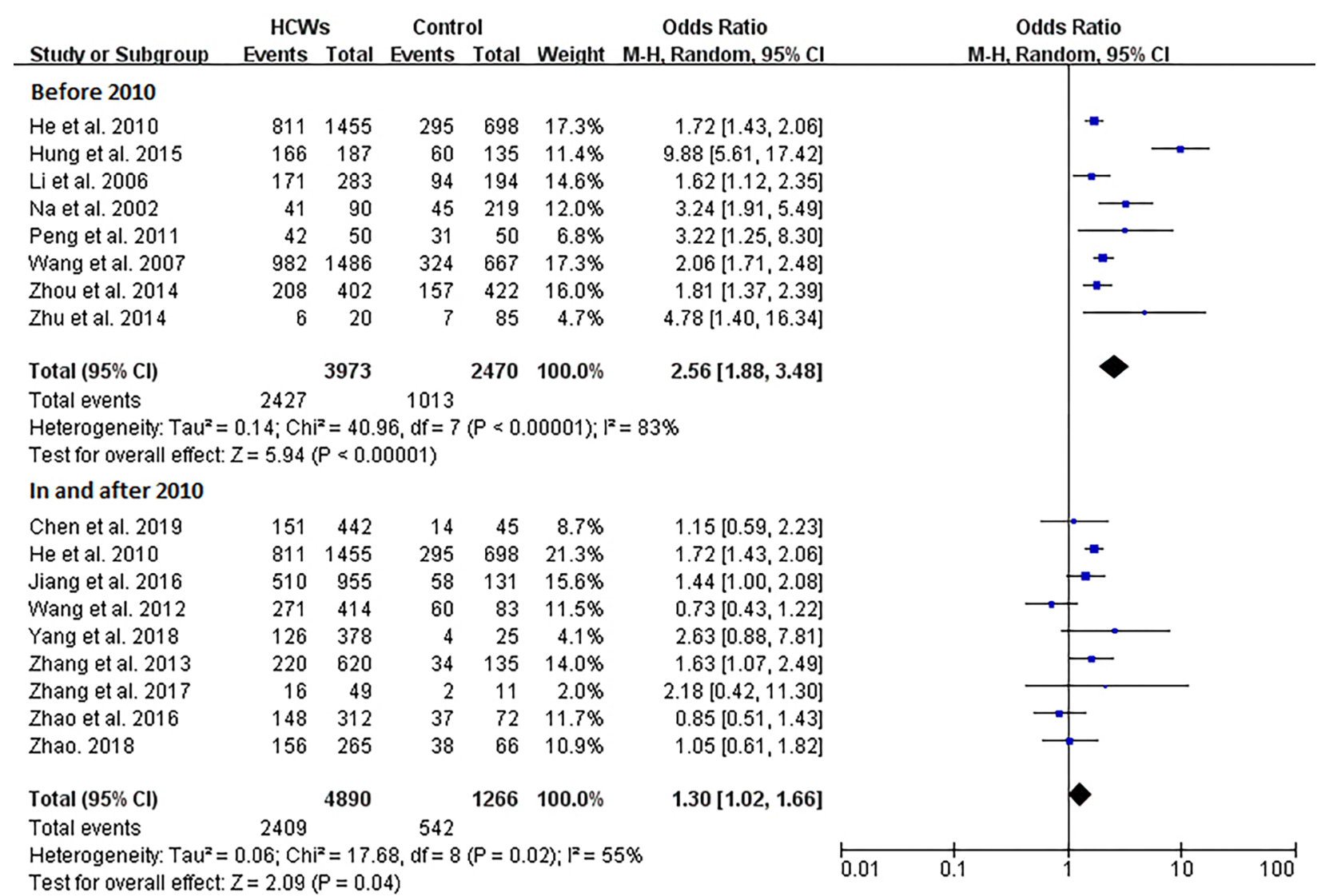

Figure S4 Forest plot showing pooled odds ratio (OR) for LTBI among HCWs according to study period. 


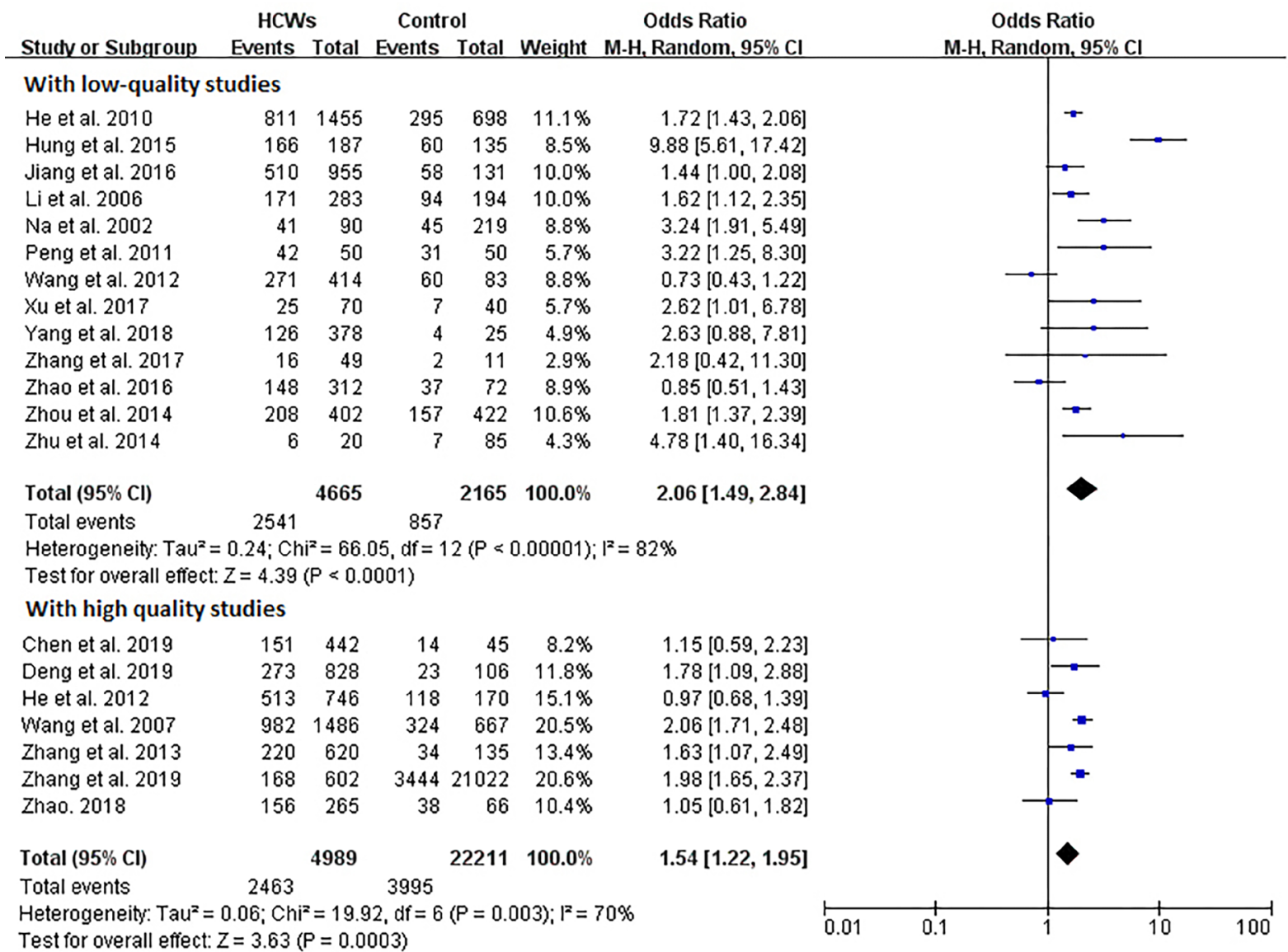

Figure S5 Forest plot showing pooled odds ratio (OR) for LTBI among HCWs according to quality of included literature. 


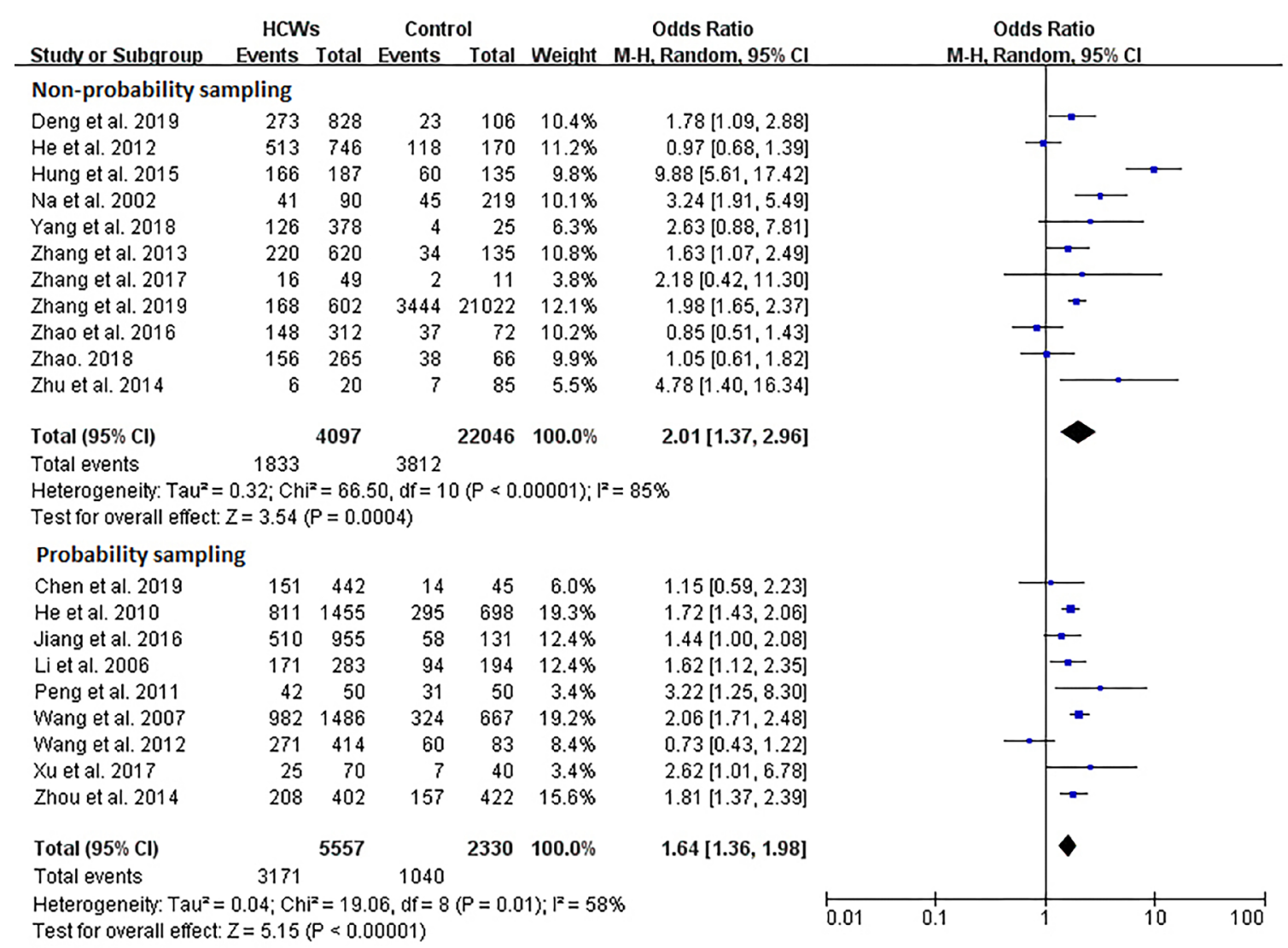

Figure S6 Forest plot showing pooled odds ratio (OR) for LTBI among HCWs according to sampling methods. 
Table S3 T-test for age difference between HCWs and control group

\begin{tabular}{|c|c|c|c|c|}
\hline Author, year, and reference & Age of HCWs $(\bar{x} \pm s)$ & Age of controls $(\bar{x} \pm s)$ & t Value & $P$ value \\
\hline Xu et al., 2017 (32) & $33.4 \pm 7.72$ & $35.9 \pm 11.4$ & 1.368 & $>0.05$ \\
\hline Zhou et al., 2014 (38) & $37.05 \pm 9.34$ & $37.82 \pm 9.54$ & 1.170 & $>0.05$ \\
\hline
\end{tabular}

Table S4 Chi-square test of gender difference between HCWs and control group

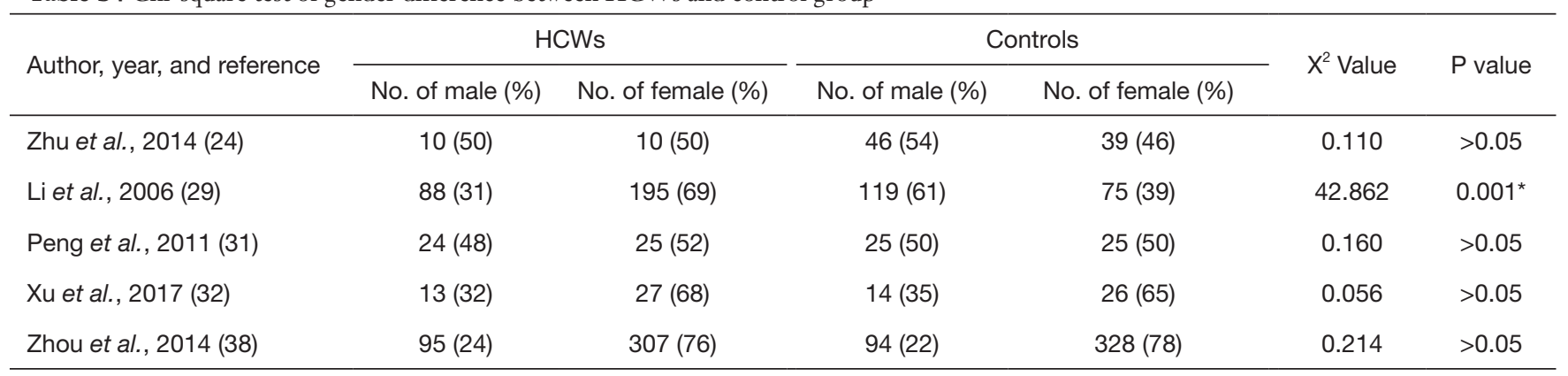

